\title{
Long-Term Follow-Up of Congenital Simple Hamartoma of the Retinal Pigment Epithelium: A Case Report
}

\author{
Yuka Ito Masahito Ohji \\ Department of Ophthalmology, Shiga University of Medical Science, Otsu, Japan
}

\section{Keywords}

Congenital simple hamartoma - Optical coherence tomography · Optical coherence tomography angiography

\begin{abstract}
Background: Congenital simple hamartoma of the retinal pigment epithelium (CSHRPE) is a rare benign tumor of the retinal pigment epithelium characterized by a focal, darkly pigmented nodule in the macular lesion in healthy persons. We report a case of CSHRPE with long-term follow-up. Case: A 41-year-old Japanese woman was incidentally discovered to have a dark lesion on the fundus of the left eye. We evaluated the patient by measuring her best-corrected visual acuity (BCVA) and by slit-lamp biomicroscopy, fundus color photography, and optical coherence tomography (OCT) over a 10-year period. The BCVA gradually declined during the early follow-up period, having decreased from 1.2 to 0.8 in the left eye 3 years after the initial examination, and then has been maintained for the following 7 years. The lesion did not show a change in OCT 10 years after the first examination. Conclusion: It is important to follow a CSHRPE carefully over the long term because visual acuity might decrease.




\section{Case Reports in Ophthalmology}

Case Rep Ophthalmol 2018;9:215-220

DOI: $10.1159 / 000487631$

(c) 2018 The Author(s). Published by S. Karger AG, Basel www.karger.com/cop

Ito and Ohji: Long-Term Follow-Up of Congenital Simple Hamartoma of the Retina Pigment Epithelium: A Case Report

\section{Introduction}

Congenital simple hamartoma of the retinal pigment epithelium (CSHRPE) is a rare benign tumor that appears as a focal, nodular, darkly pigmented lesion in the macular area in healthy persons. The lesion may involve retinal feeding vessels, vitreous pigmented cells, and retinal traction [1].

Laqua [2] reported that this tumor consisted of RPE cells in 1981, and Gass [3] classified and defined the tumor clinically as an RPE hamartoma in 1989. Shields et al. [1] reported five cases and defined the lesions as CSHRPE in 2003. There have been only a few reports of CSHRPE worldwide and, to the best of our knowledge, only two Asian cases [1, 4-14]. The previous reports noted that fluorescein angiography (FA) and optical coherence tomography (OCT) were performed to diagnose CSHRPE, whereas one report analyzed CSHRPE with OCT angiography (OCTA) [5]. There have been no reports of a clearly diagnosed CSHRPE by diagnostic OCT imaging that has been followed up for more than 3 years.

We report a patient with CSHRPE followed using the same monitors for 10 years. It also has the opportunity to measure OCTA and multifocal electroretinography (mfERG) and report the results.

\section{Case Report}

A 41-year-old Japanese woman was incidentally discovered to have a dark lesion on the fundus of her left eye by the medical checkup. She was aware of stereopsis. The decimal best-corrected visual acuity (BCVA) was 1.5 in the right eye and 1.2 in the left eye. The anterior ocular segments were normal in both eyes. The right fundus was normal. A black lesion was apparent in the foveal region, and a myelinated nerve fiber was identified in the nasal area of the left fundus (Fig. 1a). Spectral-domain OCT (Cirrus; Carl Zeiss Meditec, Inc., Dublin, CA, USA) revealed a hyperreflective lesion from the outer plexiform layer through the inner surface, protruding into the vitreous cavity, and producing deep optical shadowing (Fig. 1b). The lesion measured $345 \mu \mathrm{m}$ in horizontal scan and $160 \mu \mathrm{m}$ in vertical scan by OCT. The structures of the surrounding retina and choroid were normal, with no macular edema, retinal exudation, subretinal fluid, retinal traction, or RPE atrophy. FA has not been performed in consideration of invasion of FA because there were no significant symptoms and VA was good. After this initial evaluation, the patient had been followed without treatment. Four months later, the black lesion had not resolved, and there was no change in the OCT. Hence, we diagnosed the lesion as CSHRPE. BCVA of the left eye gradually declined from 1.2 to 0.8 for 3 years, and then was stabilized and remained the same for the following 7 years. After her 10-year follow-up, the lesion remained same, and OCT showed no change since her initial evaluation (Fig. 1d, f). FA has not been performed for 10 years because there was no change in the lesion.

In addition, fundus autofluorescence showed hypoautofluorescence at the site corresponding to the lesion (Fig. 1g). B-scan did not show any specific findings such as nodular elevation in the retina, sclera, and orbita. mfERG of both eyes showed normal patterns (Fig. 2). OCTA (Cirrus PLEX Elite 9000; Carl Zeiss Meditec, Inc., Dublin, CA, USA) showed vascular flow in the superficial plexuses (Fig. 3a), deep plexuses (Fig. 3b), outer retina (Fig. 3c), and choriocapillaris (Fig. 3d). The vascular networks appeared at the superficial and deep plexuses in the tumor, and no blood flow was detected at the outer retina and choriocapillaris in the tumor. 


\section{Case Reports in Ophthalmology}

Case Rep Ophthalmol 2018;9:215-220

DOI: 10.1159/000487631

(c) 2018 The Author(s). Published by S. Karger AG, Basel www.karger.com/cop

Ito and Ohji: Long-Term Follow-Up of Congenital Simple Hamartoma of the Retina Pigment Epithelium: A Case Report

\section{Discussion}

Because CSHRPE is asymptomatic, it is found incidentally in most cases [1, 6-8]. To the best of our knowledge, the longest period of observation of a CSHRPE was 15 years by Laqua [2]. Laqua had followed up for 15 years without OCT images [2]; however, our case had been followed with OCT images for 10 years. To the best of our knowledge, this period was the longest among past reports with OCT images.

In previously reported cases, there was no progression of the lesion, and visual acuity had not changed [1, 4, 6-13]. The boundaries of the clear black lesion in our patient, which was located in the central fovea, also did not change, although the BCVA gradually declined for the first 3 years after the initial evaluation. The BCVA, however, stabilized and remained stable for the following 7 years. Laqua reported that VA of the case was 0.3 , but there was no clear information of VA for 15 years, and the jet-black parafoveal lesion had not changed for 15 years [2].

OCT is useful for diagnosing CSHRPE as they show its characteristic findings. Shields et al. [4] were the first to analyze CSHRPE by OCT. The lesion is highly hyperreflective in the inner retina, and OCT shows complete optical shadowing. These findings were similar to those in our case. Although Laqua [2] had followed a patient with CSHRPE for 15 years, no OCT images were reported in the manuscript. Therefore, small changes over the follow-up period may be overlooked. Our case had been followed by performing OCT for 10 years and, to the best of our knowledge, this period was the longest among past reports with OCT images.

There have been reports of cases with vitreomacular traction [12] and macular edema [13]. However, there were no macular edema, retinal exudation, subretinal fluid, retinal traction, and RPE atrophy in our case. Although we do not know clearly the cause of vision loss, we think that the influence of tumor location and tumor itself cannot be completely denied. Thus, we have been following this case for a long time to see whether there is secondary change by tumor.

FA has been performed as one of the diagnoses of CSHRPE. However, we followed our patient without FA considering the side effects of FA. In previous reports, FA revealed no fluorescence during the early phase and it became hyperfluorescent without leakage in the late phase $[1,9]$. In other reports, there is no leakage during any of the phases $[6,8]$.

Recently, OCTA has been developed as a noninvasive examination, making it possible to analyze vascular networks three-dimensionally. Arjmand et al. [5] analyzed a case of CSHRPE using OCTA and showed large-caliber vessels throughout the tumor at the superficial and deep plexuses and the outer retina but no flow at the choriocapillaris. In our case, vascular networks were apparent at the superficial and deep plexuses in the tumor as in the previous report. The lesion size in Arjmand's report was larger than in our case; thus, we suppose that OCTA images were different from our case. We cannot compare the difference between FA and OCTA in this case report, but we suggest that OCTA may be helpful to diagnosis and follow-up of CSHRPE because OCTA is a noninvasive examination and can visualize blood vessels for each segment.

The mfERG yielded normal findings. We think the results of mfERG were normal because the lesion was smaller than the stimulating spot size.

In conclusion, a patient with CSHRPE has been followed for 10 years. BCVA gradually decreased for the first 3 years and was then maintained for the following 7 years, while the lesion exhibited no obvious change for 10 years. It is important to follow CSHRPE carefully over the long term because visual acuity might decrease. 


\section{Case Reports in Ophthalmology}

\begin{tabular}{l|l}
\hline DOI: $10.1159 / 000487631$ & (c) 2018 The Author(s). Published by S. Karger AG, Basel
\end{tabular}
www.karger.com/cop

Ito and Ohji: Long-Term Follow-Up of Congenital Simple Hamartoma of the Retinal Pigment Epithelium: A Case Report

Statement of Ethics

The authors have no ethical conflicts to disclose.

\section{Disclosure Statement}

The authors declare no conflict of interest.

\section{References}

1 Shields CL, Shields JA, Marr BP, Sperber DE, Gass JDM: Congenital simple hamartoma of the retinal pigment epithelium. A study of five cases. Ophthalmology 2003;110:1005-1011.

2 Laqua $\mathrm{H}$ : Tumors and tumor-like lesions of the retinal pigment epithelium. Ophthalmologica 1981;183:34-38.

3 Gass JDM: Focal congenital anomalies of the retinal pigment epithelium. Eye 1989;3:1-18.

4 Shields CL, Materin MA, Karatza EC, Shields JA: Optical coherence tomography of congenital simple hamartoma of the retinal pigment epithelium. Retina 2004;24:327-328.

5 Arjmand P, Elimimian EB, Say EAT, Shields CL: Optical coherence tomography angiography of congenital simple hamartoma of the retinal pigment epithelium. Retin Cases Brief Rep 2017, DOI: 10.1097/ICB.0000000000000596.

6 Teke MY, Ozdal PC, Batioglu F, Elgin U, Ozturk F: Congenital simple hamartoma of retinal pigment epithelium: clinical and imaging findings. Case Rep Ophthalmol Med 2012;2012:1-3.

7 Grant LW, Seth RK: Congenital simple hamartoma of the retinal pigment epithelium. Semin Ophthalmol 2014;29:183-185.

8 Thorell MR, Kniggendorf VF, Arana LA, Grandinetti AA: Congenital simple hamartoma of the retinal pigment epithelium: a case report. Arq Bras Oftalmol 2014;77:114-115.

9 Lopez JM, Guerrero P: Congenital simple hamartoma of the retinal pigment epithelium: optical coherence tomography and angiography features. Retina 2006;26:704-706.

10 Gotoh M, Yoshikawa H, Kagimoto HTS, Ishibashi T: Congenital simple hamartoma of the retinal pigment epithelium in an Asian. Jpn J Ophthalmol 2008;52:144-145.

11 Kálmán Z, Tóth J: Two cases of congenital simple hamartoma of the retinal pigment epithelium. Retin Cases Brief Rep 2009;3:283-285.

12 Barnes AC, Goldman DR, Laver NV, Duker JS: Congenital simple hamartoma of the retinal pigment epithelium: clinical, optical coherence tomography, and histopathological correlation. Eye 2014;28:765-766.

13 Bach A, Gold AS, Villegas VM, Wildner AC, Latiff A, Ehlies FJ, Murray TG: Simple hamartoma of the retinal pigment epithelium with macular edema. Optom Vis Sci 2015;92:48-50.

14 Heo WJ, Park DH, Shin JP: A case of congenital simple hamartoma of the retinal pigment epithelium and Coats' disease in the same eye. Korean J Ophthalmol 2015;29:282-283. 


\section{Case Reports in Ophthalmology} www.karger.com/cop

Ito and Ohji: Long-Term Follow-Up of Congenital Simple Hamartoma of the Retinal Pigment Epithelium: A Case Report
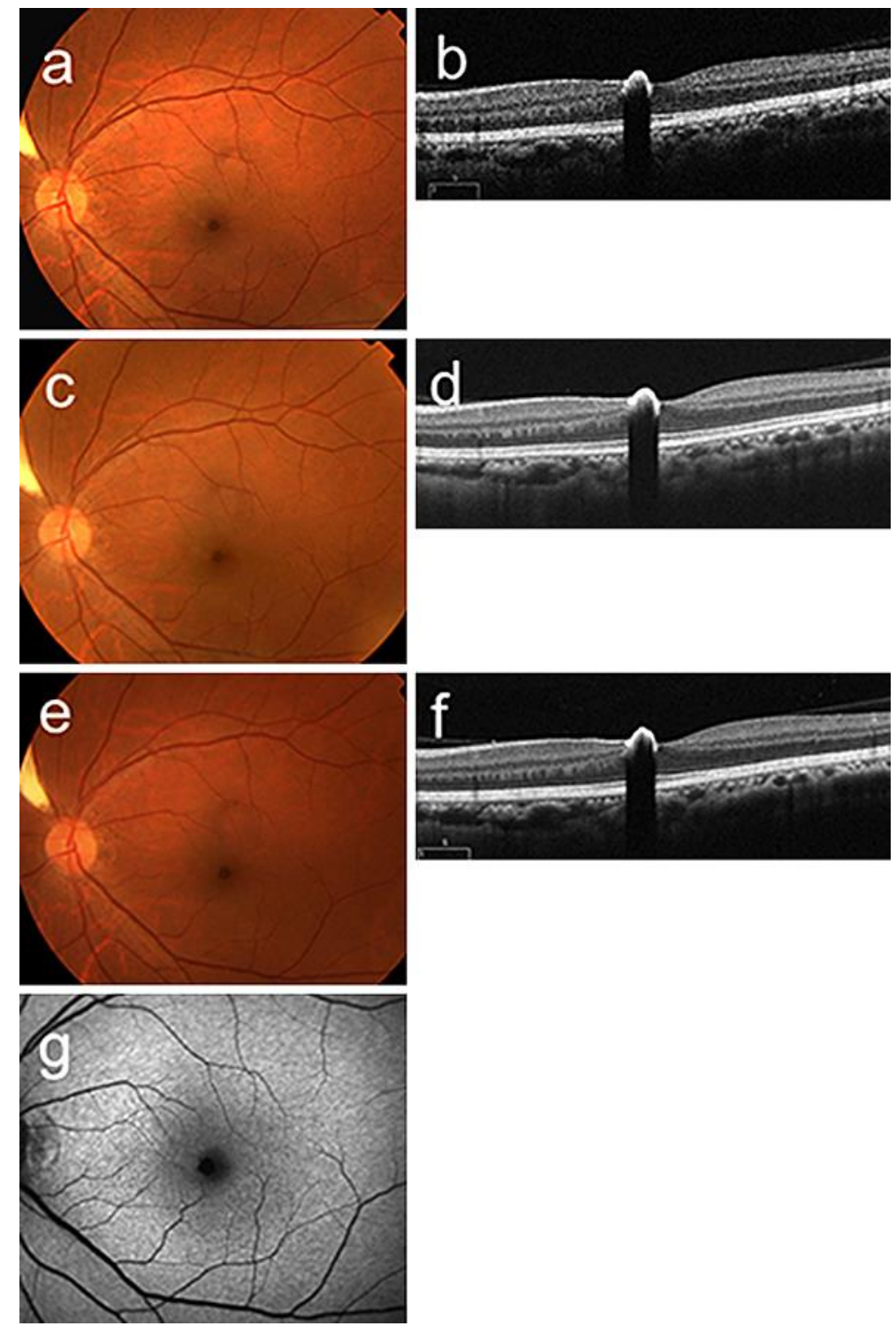

Fig. 1. Congenital simple hamartoma of retinal pigment epithelium (CSHRPE) in a 41-year-old Japanese woman. a Photograph of the fundus of the left eye at the initial examination shows a clear black lesion in the foveal region and a myelinated nerve fiber in the nasal area. b Spectral-domain optical coherence tomography (OCT) at the initial evaluation shows a hyperreflective lesion that spreads from the outer plexiform layer through the inner surface, protruding into the vitreous cavity, with deep optical shadowing at the first examination. $\mathbf{c}$ Photograph of the fundus 3 years after the first examination. $\mathbf{d}$ OCT 3 years after the first examination. e Photograph of the fundus 10 years after the first examination. $f$ OCT 10 years after the first examination $\mathbf{g}$ Fundus autofluorescence showed hypoautofluorescence at the site corresponding to the lesion. 
a
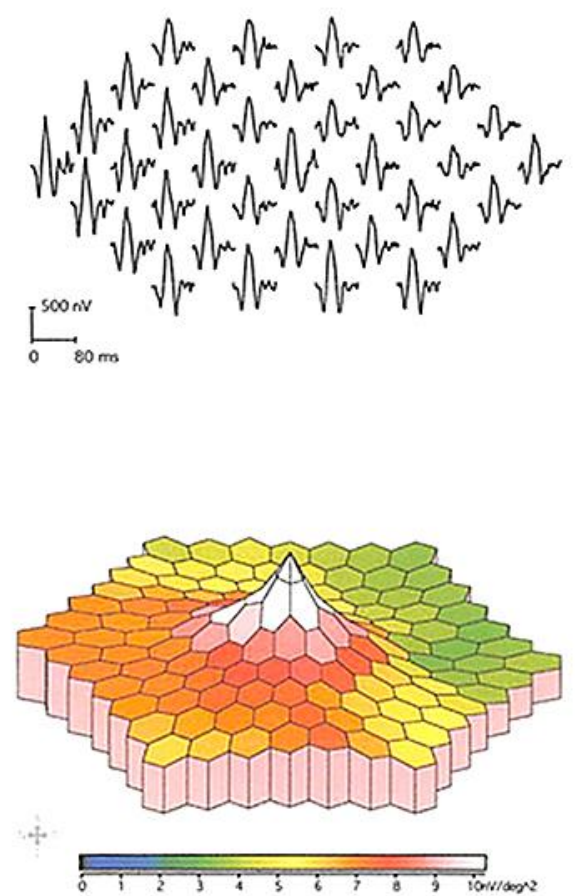

b
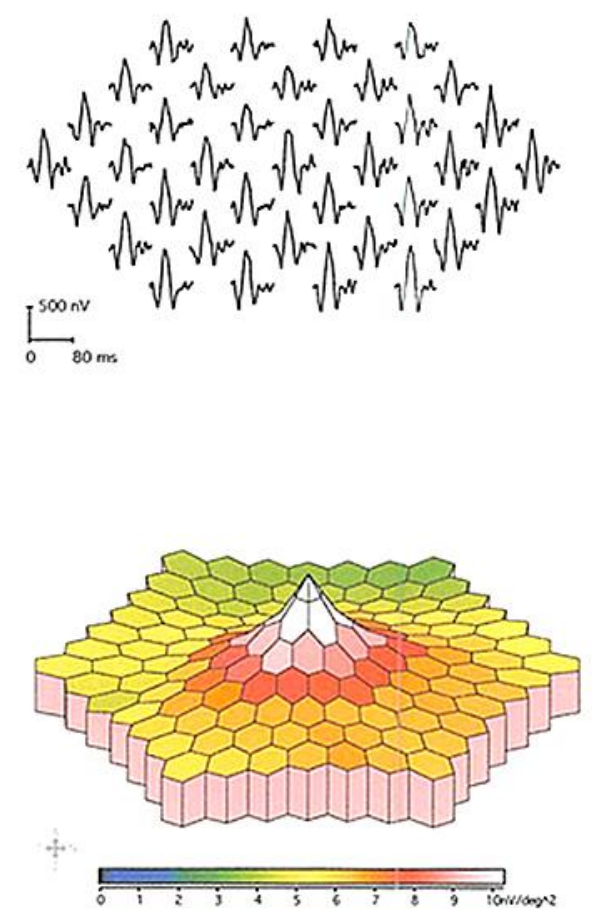

Fig. 2. Multifocal electroretinography of both eyes (a right, b left) shows normal patterns.
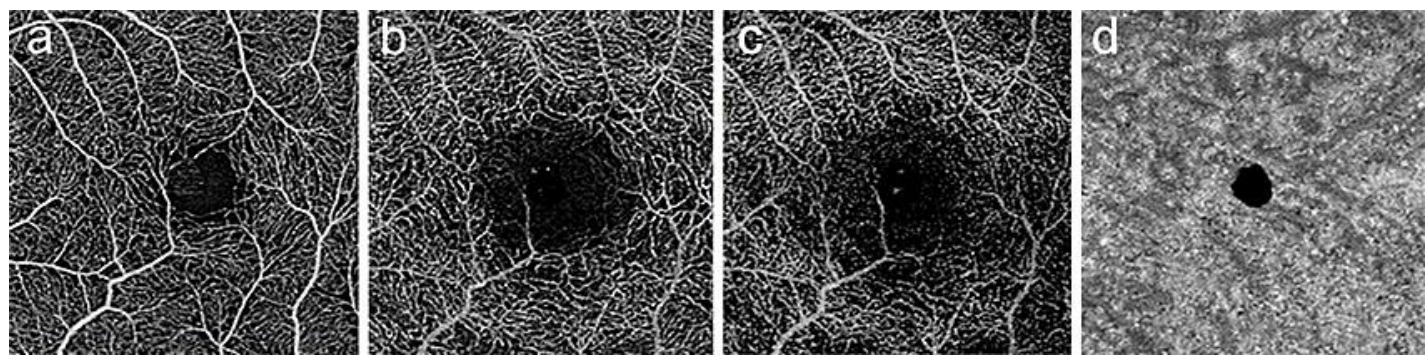

Fig. 3. Optical coherence tomography angiography was used to analyze the vascular flow of the superficial plexuses (a), deep plexuses (b), outer retina (c), and choriocapillaris (d). Vascular networks appeared at the superficial and deep plexuses. No blood flow was detected in the tumor at the outer retina or choriocapillaris. 